MATHEMATICS OF COMPUTATION

Volume 75, Number 254, Pages 711-726

S $0025-5718(05) 01813-2$

Article electronically published on December 30, 2005

\title{
SMOOTH MACRO-ELEMENTS ON POWELL-SABIN-12 SPLITS
}

\author{
LARRY L. SCHUMAKER AND TATYANA SOROKINA
}

\begin{abstract}
Macro-elements of smoothness $C^{r}$ are constructed on PowellSabin-12 splits of a triangle for all $r \geq 0$. These new elements complement those recently constructed on Powell-Sabin- 6 splits and can be used to construct convenient superspline spaces with stable local bases and full approximation power that can be applied to the solution of boundary-value problems and for interpolation of Hermite data.
\end{abstract}

\section{INTRODUCTION}

A bivariate macro-element defined on a triangle $T$ consists of a finite-dimensional linear space $\mathcal{S}$ defined on $T$, and a set $\Lambda$ of linear functionals forming a basis for the dual of $\mathcal{S}$. Usually the space $\mathcal{S}$ is chosen to be a space of polynomials or a space of piecewise polynomials defined on some subtriangulation of $T$. The members of $\Lambda$, called the degrees of freedom, are usually taken to be point evaluations of derivatives, although here we will also work with sets of linear functionals which pick off certain spline coefficients.

A macro-element defines a local interpolation scheme. In particular, if $f$ is a sufficiently smooth function, then we can define the corresponding interpolant as the unique function $s \in \mathcal{S}$ such that $\lambda s=\lambda f$ for all $\lambda \in \Lambda$. We say that a macro-element has smoothness $C^{r}$ provided that if the element is used to construct an interpolating function locally on each triangle of a triangulation $\triangle$, then the resulting piecewise function is $C^{r}$ continuous globally. Macro-elements are useful tools for building spaces of smooth splines with stable local bases and full approximation power.

Several families of $C^{r}$ macro-elements have been developed using polynomials [17, 19, and piecewise polynomials on appropriate splits; see [4, 5, 11, 12, 13, 15. and references therein. The purpose of this paper is to describe a family of $C^{r}$ macro-elements based on the Powell-Sabin-12 split; see Definition 3.1. These new macro-elements complement the existing families of $C^{r}$ macro-elements based on the Powell-Sabin-6 split [5, 9, 12, and for compatibility make use of splines of the same degrees; see however Remark 7.2, A major advantage of our new elements is that certain geometric constraints required in the Powell-Sabin-6 case can be removed; see Remark 7.4.

The paper is organized as follows. In Sect. 2 we review some well-known Bernstein-Bézier notation. Our $C^{r}$ family of macro-elements is introduced and studied in Sect. 3, while Sect. 4 contains several supporting lemmas. We discuss

Received by the editor October 29, 2004 and, in revised form, February 14, 2005.

2000 Mathematics Subject Classification. Primary 41A15, 65M60, 65N30.

Key words and phrases. Macro-elements, stable bases, spline spaces, Powell-Sabin.

The first author was supported by the Army Research Office under grant DAAD-19-99-1-0160.

(C)2005 American Mathematical Society 
the approximation power of our new macro-elements in Sect. 5. In Sect. 6 we translate our degrees of freedom into nodal functionals and discuss a related Hermite interpolation method and associated error bound. We conclude with remarks in Sect. 7 .

\section{Preliminaries}

We use Bernstein-Bézier techniques as in [1, 2, 3, 4, 15, 6, 7, 8, 9, 10, 11, 12, 13. 16, 17. In particular, we represent polynomials $p$ of degree $d$ on a triangle $T:=\left\langle v_{1}, v_{2}, v_{3}\right\rangle$ in their B-form

$$
p=\sum_{i+j+k=d} c_{i j k}^{T} B_{i j k}^{d},
$$

where $B_{i j k}^{d}$ are the Bernstein basis polynomials of degree $d$ associated with $T$. As usual, we associate the coefficients $c_{i j k}^{T}$ with the domain points $\xi_{i j k}^{T}:=\frac{\left(i v_{1}+j v_{2}+k v_{3}\right)}{d}$. We write $\mathcal{D}_{d, T}:=\left\{\xi_{i j k}^{T}\right\}_{i+j+k=d}$.

Given a triangulation $\triangle$, let $\mathcal{D}_{d, \triangle}:=\bigcup_{T \in \triangle} \mathcal{D}_{d, T}$, and let $\mathcal{S}_{d}^{0}(\triangle)$ be the space of continuous splines of degree $d$ on $\triangle$. Then it is well known that each spline in $\mathcal{S}_{d}^{0}(\triangle)$ is uniquely determined by its set of B-coefficients $\left\{c_{\xi}\right\}_{\xi \in \mathcal{D}_{d, \Delta}}$, where the coefficients of the polynomial $\left.s\right|_{T}$ are precisely $\left\{c_{\xi}\right\}_{\xi \in \mathcal{D}_{d, \Delta} \cap T}$. We recall that if $T:=\left\langle v_{1}, v_{2}, v_{3}\right\rangle$, then the ring of radius $m$ around $v_{1}$ is $R_{m}^{T}\left(v_{1}\right):=\left\{\xi_{d-m, j, k}^{T}: j+k=m\right\}$ and the disk of radius $m$ around $v_{1}$ is $D_{m}^{T}\left(v_{1}\right):=\left\{\xi_{i j k}^{T}: i \geq d-m\right\}$. If $v$ is a vertex of $\triangle$, we define the ring $R_{m}(v)$ of radius $m$ around $v$ to be the set of all domain points on rings $R_{m}^{T}(v)$ where $T$ is a triangle with vertex at $v$. The $\operatorname{disk} D_{m}(v)$ of radius $m$ around $v$ is defined similarly.

In this paper we are interested in subspaces $\mathcal{S}$ of $\mathcal{S}_{d}^{0}(\triangle)$ that satisfy additional smoothness conditions. Following 6, to describe smoothness we shall make use of smoothness functionals defined as follows. Let $T:=\left\langle v_{1}, v_{2}, v_{3}\right\rangle$ and $\widetilde{T}:=\left\langle v_{4}, v_{3}, v_{2}\right\rangle$ be two adjoining triangles that share the edge $e:=\left\langle v_{2}, v_{3}\right\rangle$, and let $c_{i j k}$ and $\tilde{c}_{i j k}$ be the coefficients of the B-representations of $s_{T}$ and $s_{\widetilde{T}}$, respectively. Then for any $n \leq m \leq d$, let $\tau_{e, m}^{n}$ be the linear functional defined on $\mathcal{S}_{d}^{0}(\triangle)$ by

$$
\tau_{e, m}^{n} s:=\tilde{c}_{n, m-n, d-m}-\sum_{i+j+k=n} c_{i, j+d-m, k+m-n} B_{i j k}^{n}\left(v_{4}\right)
$$

where $B_{i j k}^{n}$ are the Bernstein polynomials of degree $n$ on the triangle $T$. In terms of these linear functionals, the condition that $s$ be $C^{r}$ smooth across the edge $e$ is equivalent to

$$
\tau_{e, m}^{n} s=0, \quad n \leq m \leq d, 0 \leq n \leq r .
$$

Smoothness conditions can be used to directly compute coefficients of one piece of a spline from another. They can also be used in situations where some of the coefficients of two different pieces of $s$ are known. The following well-known lemma [4] (see also Lemma 3.3 of [7]) shows how this works for computing coefficients on the ring $R_{m}^{T}\left(v_{2}\right) \cup R_{m}^{\widetilde{T}}\left(v_{2}\right)$.

Lemma 2.1. Suppose $T:=\left\langle v_{1}, v_{2}, v_{3}\right\rangle$ and $\widetilde{T}:=\left\langle v_{4}, v_{3}, v_{2}\right\rangle$ are two triangles sharing an edge $e:=\left\langle v_{2}, v_{3}\right\rangle$, and suppose the points $v_{1}, v_{2}, v_{4}$ are not collinear. Let $s \in \mathcal{S}_{d}^{0}(\triangle)$, where $\triangle:=T \cup \widetilde{T}$, and suppose that $\tau_{e, m}^{n} s=0, n=\ell+1, \ldots, q+\tilde{q}-\ell$ for some $\ell, m, q, \tilde{q}$ with $0 \leq q, \tilde{q},-1 \leq \ell \leq q, \tilde{q}$, and $q+\tilde{q}-\ell \leq m \leq d$. Suppose 
that all coefficients $c_{i j k}$ and $\tilde{c}_{i j k}$ of the polynomials $\left.s\right|_{T}$ and $\left.s\right|_{\widetilde{T}}$ corresponding to domain points in $D_{m}\left(v_{2}\right)$ are known except for

$$
\begin{aligned}
c_{\nu}:=c_{\nu, d-m, m-\nu}, & \nu & =\ell+1, \ldots, q, \\
\tilde{c}_{\nu}:=\tilde{c}_{\nu, m-\nu, d-m}, & \nu & =\ell+1, \ldots, \tilde{q} .
\end{aligned}
$$

Then these coefficients are uniquely determined by the smoothness conditions.

If $s$ is a spline in $\mathcal{S}_{d}^{0}(\triangle)$ that satisfies additional smoothness conditions beyond $C^{0}$ continuity, then clearly we cannot independently choose all of its coefficients

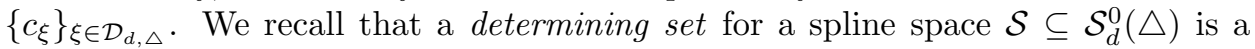
subset $\mathcal{M}$ of the set of domain points $\mathcal{D}_{d, \triangle}$ such that if we set $c_{\xi}=0$ for all $\xi \in \mathcal{M}$, then $s \equiv 0$. The set $\mathcal{M}$ is called a minimal determining set (MDS) for $\mathcal{S}$ if there is no smaller determining set. It is known that $\mathcal{M}$ is a MDS for $\mathcal{S}$ if and only if every spline $s \in \mathcal{S}$ is uniquely determined by its set of B-coefficients $\left\{c_{\xi}\right\}_{\xi \in \mathcal{M}}$.

A MDS $\mathcal{M}$ is called local provided that there is an integer $n$ such that for every $\xi \in \mathcal{D}_{d, \triangle} \cap T$ and every triangle $T$ in $\triangle, c_{\xi}$ is a linear combination of $\left\{c_{\eta}\right\}_{\eta \in \Gamma_{\xi}}$ where $\Gamma_{\xi}$ is a subset of $\mathcal{M}$ with $\Gamma_{\xi} \subset \operatorname{star}^{n}(T)$. Here $\operatorname{star}^{n}(T):=\operatorname{star}\left(\operatorname{star}^{n-1}(T)\right)$ for $n \geq 2$, where if $U$ is a cluster of triangles, $\operatorname{star}(U)$ is the set of all triangles that have a nonempty intersection with $U$. Moreover, $\mathcal{M}$ is called stable, provided that there is a constant $K$ depending on the smallest angle in $\triangle$ such that

$$
\left|c_{\xi}\right| \leq K \max _{\eta \in \Gamma_{\xi}}\left|c_{\eta}\right|, \quad \text { for all } \xi \in \mathcal{D}_{d, \triangle}
$$

A linear functional $\lambda$ defined on $\mathcal{S}_{d}^{0}(\triangle)$ is called a nodal functional provided that $\lambda s$ is a combination of values and/or derivatives of $s$ at some point $\eta$. A collection $\{\lambda\}_{\lambda \in \mathcal{N}}$ is called a nodal determining set for a spline space $\mathcal{S} \subseteq \mathcal{S}_{d}^{0}(\triangle)$ if $\lambda s=0$ for all $\lambda \in \mathcal{N}$ implies $s \equiv 0 . \mathcal{N}$ is called a nodal minimal determining set (NMDS) for $\mathcal{S}$ if there is no smaller nodal determining set.

\section{A family of $C^{r}$ Powell-Sabin-12 maCRo-Elements}

We now define the Powell-Sabin split of interest in this paper.

Definition 3.1. Given a triangle $T=\left\{v_{1}, v_{2}, v_{3}\right\}$, for each $1 \leq i \leq 3$, let $w_{i}$ be the midpoint of the edge $e_{i}:=\left\langle v_{i+1}, v_{i+2}\right\rangle$ opposite to $v_{i}$, where we set $v_{4}:=v_{1}$. Draw in the line segments $\left\langle v_{i}, w_{i}\right\rangle, i=1,2,3$. Then it is easy to see that these three line segments intersect at the barycenter $v_{T}:=\left(v_{1}+v_{2}+v_{3}\right) / 3$ of $T$. The resulting partition $T_{\mathrm{PS} 6}$ of $T$ into six triangles is called the Powell-Sabin- 6 split of $T$; see Figure 1 (left). If we now draw in the line segments $\left\langle w_{i}, w_{i+1}\right\rangle, i=1,2,3$, where $w_{4}:=w_{1}$, then the resulting partition $T_{\mathrm{PS} 12}$ of $T$ into twelve triangles is called the Powell-Sabin-12 split of $T$; see Figure 1 (right).

We need some additional notation and terminology connected with Powell-Sabin12 splits. For each $i=1,2,3$, we write $u_{i}$ for the intersection of $\left\langle w_{i+1}, w_{i+2}\right\rangle$ with $\left\langle v_{i}, v_{T}\right\rangle$. Note that the $u_{i}$ are midpoints of the edges $\left\langle w_{i+1}, w_{i+2}\right\rangle$, and are singular vertices of $T_{\mathrm{PS} 12}$; i.e., vertices that are formed by two crossing lines. We refer to the edges of the form $\left\langle v_{i}, u_{i}\right\rangle$ as type-1 edges, to edges of the form $\left\langle w_{i}, v_{T}\right\rangle$ as type-2 edges, and to edges of the form $\left\langle u_{i}, v_{T}\right\rangle$ as type-3 edges.

Given a triangulation $\triangle$ of a domain $\Omega$, we write $\mathcal{V}$ and $\mathcal{E}$ for the sets of vertices and edges of $\triangle$. To define our macro-element spaces, we shall work with the refinement $\triangle_{\mathrm{PS} 12}$ of $\triangle$, which is obtained by applying the Powell-Sabin-12 split to each triangle of $\triangle$. We write $\mathcal{W}$ for the set of midpoints of edges of $\triangle$. For 

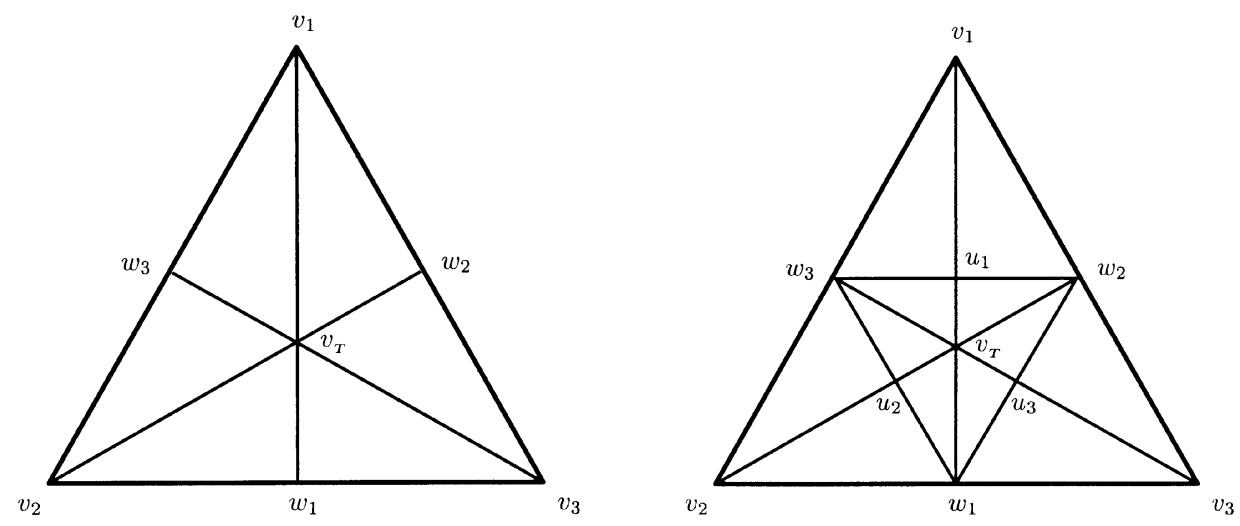

Figure 1. The Powell-Sabin-6 and Powell-Sabin-12 splits

$i=1,2,3$, we write $\mathcal{E}_{i}$ for the set of edges of $\triangle_{\mathrm{PS} 12}$ of type $i$. Let $\widetilde{\mathcal{E}}_{2}$ be a subset of $\mathcal{E}_{2}$ obtained by selecting exactly one edge of $\mathcal{E}_{2}$ for each macro-triangle in $\triangle$. As usual in spline theory, $m_{+}$is defined to be $m$ if $m>0$, and is zero otherwise.

We now introduce the spline spaces of interest in this paper. The definition depends on the value of $r$ mod 4. Given $r>0$, we define the $C^{r}$ Powell-Sabin-12 macro-element space to be

$$
\begin{aligned}
\mathcal{S}_{r}\left(\triangle_{\mathrm{PS} 12}\right) & :=\left\{s \in \mathcal{S}_{d}^{r}\left(\triangle_{\mathrm{PS} 12}\right): s \in C^{\rho}(v) \text { all } v \in \mathcal{V},\right. \\
& s \in C^{\mu}(w) \text { all } w \in \mathcal{W}, \\
& \left.\tau s=0 \text { for all } \tau \in \mathcal{T}_{1} \cup \mathcal{T}_{2}\right\},
\end{aligned}
$$

where for all $\ell \geq 0$,

\begin{tabular}{||c|c|c|c||}
\hline$r$ & $\rho$ & $\mu$ & $d$ \\
\hline $4 \ell+1$ & $6 \ell+1$ & $6 \ell+1$ & $9 \ell+2$ \\
$4 \ell+2$ & $6 \ell+3$ & $6 \ell+3$ & $9 \ell+5$ \\
$4 \ell+3$ & $6 \ell+4$ & $6 \ell+5$ & $9 \ell+7$ \\
$4 \ell+4$ & $6 \ell+6$ & $6 \ell+7$ & $9 \ell+10$ \\
\hline
\end{tabular}

$$
\begin{aligned}
& \mathcal{T}_{1}:= \begin{cases}\bigcup_{e \in \mathcal{E}_{1}}\left\{\tau_{e, \rho+j}^{r+i}\right\}_{i=1, j=1}^{2 \ell-2(\ell-j+1)_{+}, d-\rho}, & \text { if } r \text { is odd } \\
\bigcup_{e \in \mathcal{E}_{1}}\left\{\tau_{e, \rho+j}^{r+i}\right\}_{i=1, j=1}^{2 \ell+1-2(\ell-j+1)_{+}, d-\rho}, & \text { otherwise }\end{cases} \\
& \mathcal{T}_{2}:= \begin{cases}\bigcup_{e \in \mathcal{E}_{2}}\left\{\tau_{e, \mu+j}^{r+i}\right\}_{i=1, j=2}^{2 j-2, \ell}, & r=4 \ell+1, \\
\bigcup_{e \in \mathcal{E}_{2}}\left\{\tau_{e, \mu+j}^{r+i}\right\}_{i=1, j=1}^{2 j-1, \ell} \cup \bigcup_{e \in \widetilde{\mathcal{E}}_{2}}\left\{\tau_{e, \mu+\ell+1}^{r+1}\right\} \cup \bigcup_{e \in \mathcal{E}_{3}}\left\{\tau_{e, r+j}^{r+1}\right\}_{j=1}^{\ell}, & r=4 \ell+2, \\
\bigcup_{e \in \mathcal{E}_{2}}\left\{\tau_{e, \mu+j}^{r+i}\right\}_{i=1, j=1}^{2 j, \ell}, & r=4 \ell+3, \\
\bigcup_{e \in \mathcal{E}_{2}}\left\{\tau_{e, \mu+j}^{r+i}\right\}_{i=1, j=1}^{2 j+1, \ell} \cup \bigcup_{e \in \widetilde{\mathcal{E}}_{2}}\left\{\tau_{e, \mu+\ell+1}^{r+1}\right\} \cup \bigcup_{e \in \mathcal{E}_{3}}\left\{\tau_{e, r+j}^{r+1}\right\}_{j=1}^{\ell+1}, & r=4 \ell+4\end{cases}
\end{aligned}
$$

Let $n_{V}$ and $n_{E}$ be the numbers of vertices and edges of $\triangle$, respectively. For each $v \in \mathcal{V}$, let $T_{v}$ be some triangle in $\triangle_{\mathrm{PS} 12}$ with vertex at $v$. For each $e:=\left\langle v_{1}, v_{2}\right\rangle$ of $\triangle$, let $v_{T_{e}}$ be the barycenter of a triangle $T_{e}$ in $\triangle$ that contains $e$, and let $T_{e}^{1}:=\left\langle u_{1}, v_{1}, w_{e}\right\rangle$ and $T_{e}^{2}:=\left\langle u_{2}, w_{e}, v_{2}\right\rangle$ be the two subtriangles of $T_{e}$ sharing the edge $e$, where $w_{e}$ is the midpoint of $e$. In addition, let $T_{e}^{3}:=\left\langle v_{T_{e}}, w_{e}, u_{2}\right\rangle$ be one of the triangles in $\triangle_{\mathrm{PS} 12}$ containing the edge $\left\langle w_{e}, v_{T_{e}}\right\rangle$; see Figure 1 (right). 
Theorem 3.2. For all $r \geq 1$,

$$
\operatorname{dim} \mathcal{S}_{r}\left(\triangle_{\mathrm{PS} 12}\right)=\left(\begin{array}{c}
\rho+2 \\
2
\end{array}\right) n_{V}+\left[\frac{(\mu+1)^{2}}{4}+\ell(\ell+1)\right] n_{E} .
$$

Moreover, the set

$$
\mathcal{M}:=\bigcup_{v \in \mathcal{V}} \mathcal{M}_{v} \cup \bigcup_{e \in \mathcal{E}}\left(\mathcal{M}_{e}^{1} \cup \mathcal{M}_{e}^{2} \cup \mathcal{M}_{e}^{3}\right)
$$

is a stable local minimal determining set for $\mathcal{S}_{r}\left(\triangle_{\mathrm{PS} 12}\right)$, where

$$
\begin{aligned}
\text { 1) } \mathcal{M}_{v} & :=D_{\rho}(v) \cap T_{v}, \\
\text { 2) } \mathcal{M}_{e}^{1}: & :=\bigcup_{i=1}^{\ell}\left\{\xi_{\rho+\mu-d+i+1, d-\rho-j, d-\mu-i+j-1}^{T_{e}^{1}}\right\}_{j=1}^{i}, \\
\text { 3) } \mathcal{M}_{e}^{2}: & =\bigcup_{i=1}^{\ell}\left\{\xi_{\rho+\mu-d+i+1, d-\mu-i+j-1, d-\rho-j}^{T_{e}^{2}}\right\}_{j=1}^{i}, \\
\text { 4) } \mathcal{M}_{e}^{3}: & :=\bigcup_{j=0}^{(\mu-1) / 2}\left\{\xi_{i+j, d-i-2 j, j}^{T_{e}^{3}}\right\}_{i=1}^{\mu-2 j} .
\end{aligned}
$$

Proof. To show that $\mathcal{M}$ is a stable local minimal determining set, we show that we can set the coefficients $\left\{c_{\xi}\right\}_{\xi \in \mathcal{M}}$ of a spline in $\mathcal{S}_{r}\left(\triangle_{\mathrm{PS} 12}\right)$ to arbitrary values, and that all other coefficients of $s$ are then uniquely, locally, and stably determined. First, for each $v \in \mathcal{V}$, we set the coefficients corresponding to $\mathcal{M}_{v}$. Then using the $C^{\rho}$ smoothness at $v$, we can uniquely compute the coefficients of $s$ corresponding to all other domain points in $D_{\rho}(v)$. This is a stable local process.

At this point it is not obvious that the coefficients that we have determined so far are compatible with each other since they may be connected by smoothness conditions. Indeed, for any two vertices $u$ and $v$ that are connected by an edge of $\triangle$, there exist chains of smoothness conditions that involve coefficients in both of the disks $D_{\rho}(u)$ and $D_{\rho}(v)$ along with other yet undetermined coefficients. As we progress we have to be sure that as we compute these undetermined coefficients, all of these smoothness conditions are verified.

For each $e:=\langle u, v\rangle \in \mathcal{E}$, we now apply Lemma 4.1 to determine the coefficients of $s$ corresponding to domain points in the disk $D_{\mu}\left(w_{e}\right)$, where $w_{e}$ is the midpoint of $e$. Due to the $C^{\mu}$ smoothness at $w_{e}$, we can regard the coefficients of $s$ in this disk as coefficients of a polynomial $g$ of degree $\mu$. The lemma insures that we can set the coefficients of $s$ corresponding to the domain points in $\mathcal{M}_{e}^{3}$ to arbitrary values, and that all coefficients corresponding to the remaining domain points in $D_{\mu}\left(w_{e}\right)$ are uniquely and stably determined. Since the lemma allows arbitrary values for the coefficients corresponding to domain points in the sets $D_{\rho}(u) \cap D_{\mu}\left(w_{e}\right)$ and $D_{\rho}(v) \cap D_{\mu}\left(w_{e}\right)$, it follows that all smoothness conditions connecting coefficients associated with domain points in $\left[D_{\rho}(u) \cup D_{\rho}(v)\right] \cap D_{\mu}\left(w_{e}\right)$ are satisfied; i.e., there are no incompatibilities due to these smoothness conditions. We still have to watch for possible incompatibilities due to other smoothness conditions involving domain points outside of the disks $\left\{D_{\rho}(v)\right\}_{v \in \mathcal{V}}$ and $\left\{D_{\mu}(w)\right\}_{w \in \mathcal{W}}$.

Our next step is to set the coefficients corresponding to the sets $\mathcal{M}_{e}^{1}$ and $\mathcal{M}_{e}^{2}$ for each edge $e$ of $\triangle$. If $e:=\left\langle v_{1}, v_{2}\right\rangle$ is an interior edge of $\triangle$ with midpoint $w_{e}$, then using the $C^{r}$ smoothness conditions across the edge $e$, we can uniquely determine 
the coefficients corresponding to the domain points in the sets

$$
\begin{aligned}
\widetilde{\mathcal{M}}_{e}^{1} & :=\bigcup_{i=1}^{\ell}\left\{\xi_{\rho+\mu-d+i+1, d-\rho-j, d-\mu-i+j-1}^{\widetilde{T}_{e}^{1}}\right\}_{j=1}^{i}, \\
\widetilde{\mathcal{M}}_{e}^{2} & :=\bigcup_{i=1}^{\ell}\left\{\xi_{\rho+\mu-d+i+1, d-\mu-i+j-1, d-\rho-j}^{\widetilde{T}_{e}^{2}}\right\}_{j=1}^{i},
\end{aligned}
$$

where $\widetilde{T}_{e}^{1}:=\left\langle\tilde{u}_{1}, v_{1}, w_{e}\right\rangle$ and $\widetilde{T}_{e}^{2}:=\left\langle\tilde{u}_{2}, w_{e}, v_{2}\right\rangle$ are the triangles in $\triangle_{\mathrm{PS} 12}$ that share edges with $T_{e}^{1}$ and $T_{e}^{2}$, respectively. At this point we have made sure that all smoothness conditions up to order $r$ across $e$ are satisfied.

For each type-1 edge $e:=\langle v, u\rangle$, we now show how to use Lemma 2.1 to compute coefficients on the rings $R_{\rho+j}(v)$ for $j=1, \ldots, d-\rho$. Fix $1 \leq j \leq d-\rho$. Then it is easy to see that there are exactly $n:=2(d-\mu)-1-2(\ell-j+1)_{+}$unset coefficients on $R_{\rho+j}(v)$. Now combining the $C^{r}$ smoothness conditions across $e$ with the special conditions in $\mathcal{T}_{1}$ associated with this edge gives us a set of exactly $n$ (univariate) smoothness conditions that uniquely determine these coefficients; see Lemma 2.1. By the geometry, the matrix of this nonsingular $n \times n$ linear system is the same for all edges $e \in \mathcal{E}_{1}$, and thus the computation is stable in the sense that (2.3) holds.

We now show that the coefficients corresponding to the remaining domain points are also uniquely determined while maintaining all smoothness conditions. These remaining domain points lie inside triangles of the form $T:=\left\langle w_{1}, w_{2}, w_{3}\right\rangle$, where the $w_{i} \in \mathcal{W}$. Let $T_{\mathrm{PS} 6}$ be the Powell-Sabin-6 split of $T$; see Figure 4 . We have already determined all coefficients corresponding to domain points in the disks $D_{\mu}\left(w_{i}\right)$ for $i=1,2,3$. In addition, by the $C^{r}$ smoothness across the edges $e_{i}:=\left\langle w_{i}, w_{i+1}\right\rangle$ for $i=1,2,3$, the coefficients corresponding to domain points on the rings $R_{d-j}\left(v_{T}\right)$ for $j=0, \ldots, r$ are also determined. For each $i=1,2,3$, the fact that the midpoint $u_{i-1}$ of $e_{i}$ is a singular vertex insures that all $C^{r}$ smoothness conditions across the edge $\left\langle u_{i-1}, v_{T}\right\rangle$ are automatically satisfied, and there are no incompatibilities. Now we can apply Lemma 4.2 to uniquely and stably determine all coefficients of $s$ corresponding to the remaining domain points in $T$. We have shown that $\mathcal{M}$ is a stable local minimal determining set for $\mathcal{S}_{r}\left(\triangle_{\mathrm{PS} 12}\right)$.

To complete the proof, we note that the dimension of $\mathcal{S}_{r}\left(\triangle_{\mathrm{PS} 12}\right)$ is equal to the cardinality of $\mathcal{M}$, which is easily seen to be the number in (3.2).

TABLE 1 . The dimension of $\mathcal{S}_{r}\left(T_{\mathrm{PS} 12}\right)$

\begin{tabular}{||r|r|r|r|r||}
\hline$r$ & $\rho$ & $\mu$ & $d$ & $\operatorname{dim}$ \\
\hline 1 & 1 & 1 & 2 & 12 \\
2 & 3 & 3 & 5 & 42 \\
3 & 4 & 5 & 7 & 72 \\
4 & 6 & 7 & 10 & 132 \\
5 & 7 & 7 & 11 & 162 \\
6 & 9 & 9 & 14 & 246 \\
7 & 10 & 11 & 16 & 312 \\
8 & 12 & 13 & 19 & 426 \\
9 & 13 & 13 & 20 & 480 \\
10 & 15 & 15 & 23 & 618 \\
11 & 16 & 17 & 25 & 720 \\
12 & 18 & 19 & 28 & 888 \\
\hline
\end{tabular}



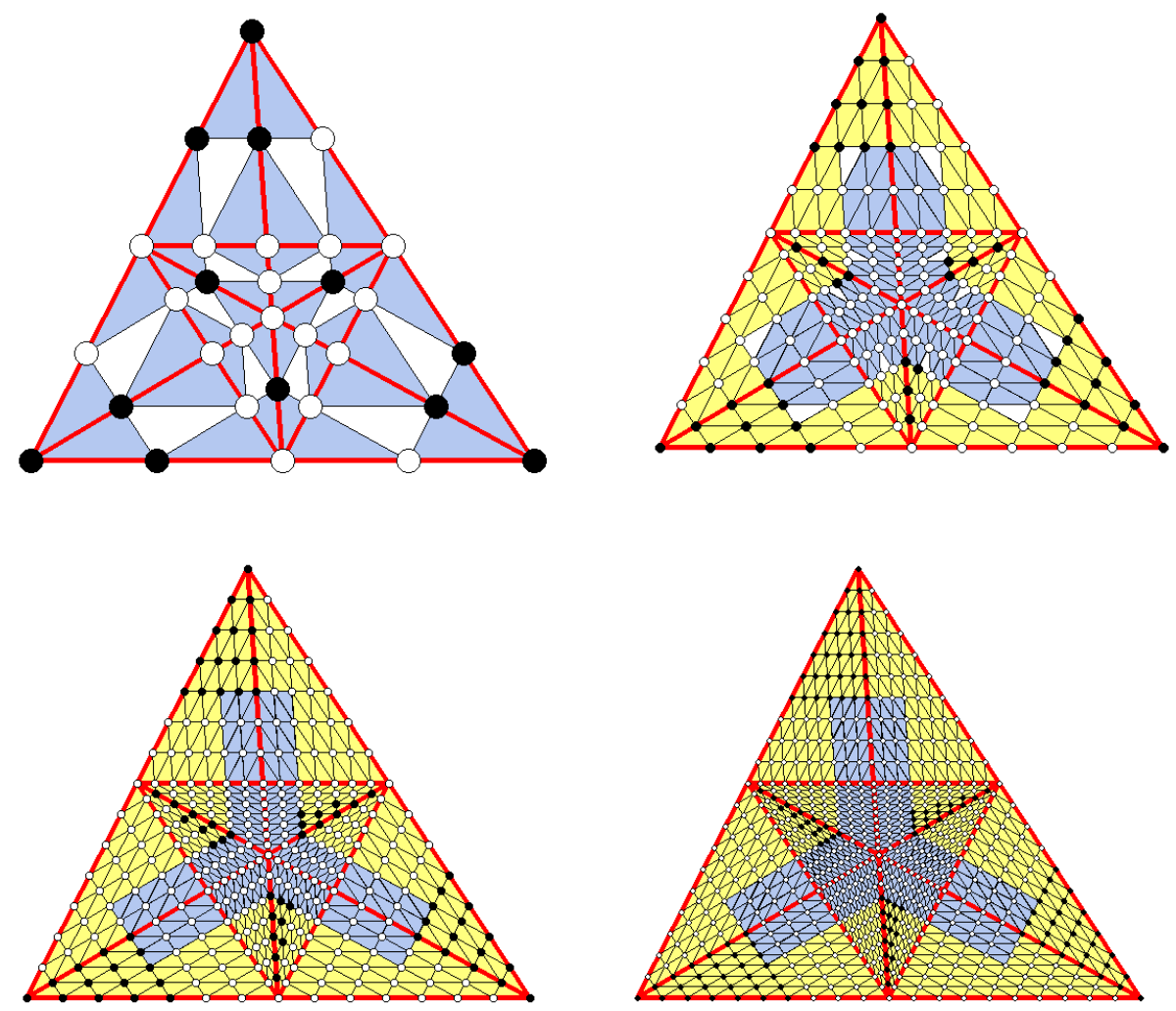

Figure 2. Minimal determining sets for $\mathcal{S}_{r}\left(T_{\mathrm{PS} 12}\right)$ for $r=1,2,3,4$

For the Powell-Sabin-12 split $T_{\mathrm{PS} 12}$ of a single triangle, Table 1 shows the values of $r, \rho, \mu, d$ and $\operatorname{dim} \mathcal{S}_{r}\left(T_{\mathrm{PS} 12}\right)$ for $1 \leq r \leq 12$. Figure 2 shows the corresponding minimal determining sets for $r=1,2,3,4$, where the points in $\mathcal{M}$ are marked with black dots.

\section{Two Lemmas}

In this section we establish two lemmas which are needed for the proof of Theorem 3.2. Our first lemma concerns a special MDS for the space of polynomials $\mathcal{P}_{\mu}$ in the case where $\mu$ is odd.

Lemma 4.1. Let $\triangle$ be the triangulation shown in Figure 3 with six vertices $v_{1}, \ldots$, $v_{6}$, where we suppose that $v_{4}$ is the midpoint of the edge $e:=\left\langle v_{3}, v_{5}\right\rangle$. Let $T:=$ $\left\langle v_{1}, v_{4}, v_{6}\right\rangle$. Suppose $\mu$ is odd, and let $m:=\frac{\mu-1}{2}$. Let $\mathcal{M}:=D_{m}\left(v_{3}\right) \cup D_{m}\left(v_{5}\right) \cup \mathcal{M}_{e} \subset$ $\mathcal{D}_{\triangle, \mu}$, where

$$
\mathcal{M}_{e}:=\bigcup_{j=0}^{m}\left\{\xi_{i+j, \mu-i-2 j, j}^{T}\right\}_{i=1}^{\mu-2 j} .
$$

Then $\mathcal{M}$ is a stable minimal determining set for $\mathcal{P}_{\mu}$. 


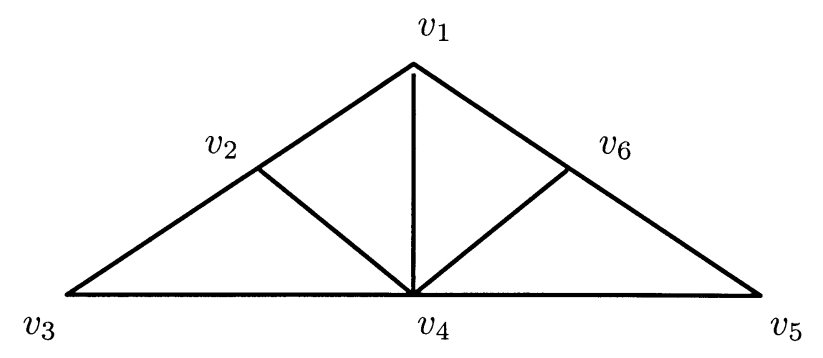

Figure 3 . The triangulation of Lemma 4.1

Proof. It is easy to check that $\# \mathcal{M}=\left(\begin{array}{c}\mu+2 \\ 2\end{array}\right)=\operatorname{dim} \mathcal{P}_{\mu}$, and thus it suffices to prove that if we set the coefficients of $s \in \mathcal{P}_{\mu}$ corresponding to $\xi \in \mathcal{M}$, then all other coefficients are stably determined. To this end, we consider the B-representation of $\tilde{s} \equiv s$ relative to the triangulation $\widetilde{\triangle}$ consisting of the two triangles $\widetilde{T}_{1}:=\left\langle v_{1}, v_{3}, v_{4}\right\rangle$ and $\widetilde{T}_{2}:=\left\langle v_{1}, v_{4}, v_{5}\right\rangle$. We denote the corresponding coefficients of $\tilde{s}$ by $\tilde{c}_{\eta}$ for $\eta \in \mathcal{D}_{\mu, \widetilde{\triangle}}$. The values of $c_{\xi}$ for $\xi \in D_{m}\left(v_{3}\right) \cap \mathcal{D}_{\mu, \triangle}$ stably determine all derivatives of $s$ up to order $m$ at $v_{3}$, which in turn stably determine the coefficients $\tilde{c}_{\eta}$ for all $\eta \in D_{m}\left(v_{3}\right) \cap \mathcal{D}_{\mu, \widetilde{\triangle}}$. A similar argument shows that $\tilde{c}_{\eta}$ are stably determined for all $\eta \in D_{m}\left(v_{5}\right) \cap \mathcal{D}_{\mu, \widetilde{\Delta}}$.

We now claim that all coefficients of $\tilde{s}$ corresponding to domain points in the set

$$
\widetilde{\mathcal{M}}_{e}:=\bigcup_{j=0}^{m}\left\{\eta_{i+j, \mu-i-2 j, j}^{\widetilde{T}_{2}}\right\}_{i=1}^{\mu-2 j}
$$

are stably determined from the coefficients $\left\{c_{\xi}\right\}_{\xi \in \mathcal{M}_{e}}$. To see this, note that since $v_{6}$ lies on the edge $\left\langle v_{1}, v_{5}\right\rangle$, the barycentric coordinates of $v_{5}$ relative to $T$ have the form $\left(b_{1}, 0, b_{2}\right)$ with $b_{1}+b_{2}=1$. Then using the de Casteljau algorithm to convert the B-coefficients of $s$ relative to $T$ into B-coefficients of $\tilde{s}$ relative to $\widetilde{T}_{2}$, we find that for each $1 \leq i \leq \mu-2 j$ and $0 \leq j \leq m$, the coefficient of $\tilde{s}$ corresponding to $\eta_{i+j, \mu-i-2 j, j}^{\widetilde{T}_{2}}$ is a stable linear combination of the coefficients $\left\{c_{\xi}\right\}_{\xi \in \mathcal{M}_{e}}$.

It is easy to check that $D_{m}^{\widetilde{T}_{2}}\left(v_{1}\right) \cap \mathcal{D}_{\mu, \widetilde{\triangle}} \subset \widetilde{\mathcal{M}}_{e}$. Then using the smoothness across the edge $\left\langle v_{1}, v_{4}\right\rangle$ of $\widetilde{\triangle}$, we can stably compute the coefficients of $\tilde{s}$ corresponding to the remaining domain points in $D_{m}\left(v_{1}\right) \cap \mathcal{D}_{\mu, \widetilde{\triangle}}$. We have now determined all coefficients of $\tilde{s}$ except for those corresponding to $\mu-j$ domain points on $R_{\mu-j}\left(v_{1}\right) \cap$ $\mathcal{D}_{\mu, \widetilde{\triangle}}$ for each $j=0, \ldots, m$. Since the coefficients associated with $R_{\mu-j}\left(v_{1}\right)$ are subjected to precisely $\mu-j$ (univariate) smoothness conditions across the edge $\left\langle v_{4}, v_{1}\right\rangle$, we can use Lemma 2.1 to stably compute them. Finally, to complete the proof, we note that the coefficients $c_{\xi}$ of $s$ can now be stably computed from those of $\tilde{s}$ by subdivision.

Our second lemma deals with splines on the Powell-Sabin- 6 split $T_{\mathrm{PS} 6}$ of a single triangle. Since we want to apply this lemma to the triangle $T:=\left\langle w_{1}, w_{2}, w_{3}\right\rangle$ that is inside the Powell-Sabin-12 split shown in Figure 1 (right), we label its vertices as in Figure 4, where we assume $u_{i}$ is the midpoint of the edge opposite $w_{i}$ for $i=1,2,3$, and $v_{T}:=\left(w_{1}+w_{2}+w_{3}\right) / 3$ is the barycenter of $T$. As in Sect. 3, we write $\mathcal{E}_{2}$ for the set of edges of $T_{\mathrm{PS} 6}$ of the form $\left\langle w_{i}, v_{T}\right\rangle$, and $\mathcal{E}_{3}$ for the set of edges of $T_{\mathrm{PS} 6}$ of the form $\left\langle u_{i}, v_{T}\right\rangle$. Given $r, \mu$, and $d$ as in (3.1), let $\mathcal{T}_{2}$ be the 


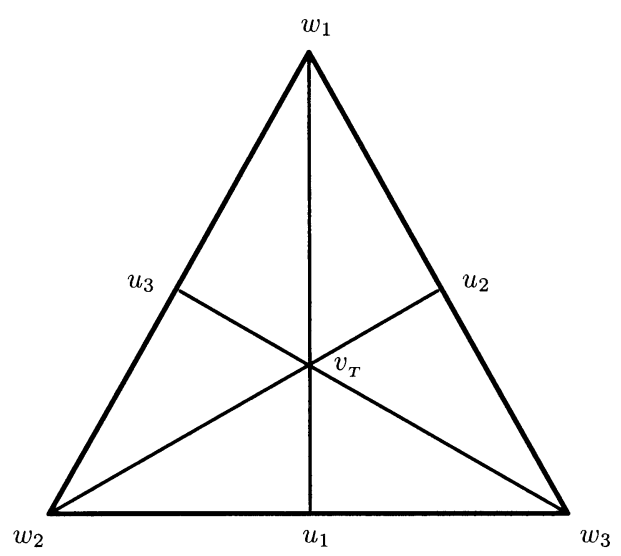

Figure 4. The labelling of the Powell-Sabin-6 split for Lemma 4.2

corresponding set of special smoothness conditions defined there. Then we consider the following superspline space:

$$
\begin{array}{r}
\mathcal{S}_{r}\left(T_{\mathrm{PS} 6}\right):=\left\{s \in \mathcal{S}_{d}^{r}\left(T_{\mathrm{PS} 6}\right): s \in C^{\mu}\left(w_{i}\right) \text { all } i=1,2,3,\right. \\
\left.\tau s=0 \text { for all } \tau \in \mathcal{T}_{2}\right\} .
\end{array}
$$

For each $n=1,2,3$, let $T_{n}:=\left\langle v_{T}, w_{n}, u_{n+2}\right\rangle$.

Lemma 4.2. For all $r \geq 1$,

$$
\operatorname{dim} \mathcal{S}_{r}\left(T_{\mathrm{PS} 6}\right)=3\left(\begin{array}{c}
\mu+2 \\
2
\end{array}\right)+3(2 d-2 \mu-r-1)(r+1) .
$$

Moreover, the set

$$
\mathcal{M}:=\bigcup_{n=1}^{3}\left(\mathcal{M}_{w_{n}} \cup \mathcal{M}_{u_{n}}\right)
$$

is a stable minimal determining set, where $\mathcal{M}_{w_{n}}:=D_{\mu}\left(w_{n}\right) \cap T_{n}$ and

$$
\mathcal{M}_{u_{n}}:= \begin{cases}\bigcup_{j=1}^{2 \ell}\left\{\xi_{i, d-\mu-j, \mu-i+j}^{T_{n}}\right\}_{i=0}^{r}, & \text { if } r \text { is odd }, \\ \bigcup_{j=1}^{2 \ell+1}\left\{\xi_{i, d-\mu-j, \mu-i+j}^{T_{n}}\right\}_{i=0}^{r}, & \text { otherwise. }\end{cases}
$$

Proof. We show below that $\mathcal{M}$ is a determining set, and thus $\operatorname{dim} \mathcal{S}_{r}\left(T_{\mathrm{PS} 6}\right) \leq \# \mathcal{M}$. It is easily seen that

$$
\# \mathcal{M}= \begin{cases}78 \ell^{2}+57 \ell+9, & r=4 \ell+1 \\ 78 \ell^{2}+111 \ell+39, & r=4 \ell+2 \\ 78 \ell^{2}+141 \ell+63, & r=4 \ell+3 \\ 78 \ell^{2}+195 \ell+123, & r=4 \ell+4\end{cases}
$$

which is equal to the expression in Lemma 4.2. We now derive a lower bound for $\operatorname{dim} \mathcal{S}_{r}\left(T_{\mathrm{PS} 6}\right)$. By Theorem 2.2 of [16],

$$
\operatorname{dim} \mathcal{S}_{d}^{r}\left(T_{\mathrm{PS} 6}\right)=\left(\begin{array}{c}
r+2 \\
2
\end{array}\right)+6\left(\begin{array}{c}
d-r+1 \\
2
\end{array}\right)+\sigma
$$


where

$$
\sigma= \begin{cases}\left(r^{2}-1\right) / 4, & \text { if } r \text { is odd } \\ r^{2} / 4, & \text { if } r \text { is even }\end{cases}
$$

Enforcing the $C^{\mu}$ continuity at the vertices $w_{1}, w_{2}, w_{3}$ of $T$ requires $3\left(\begin{array}{c}\mu-r+1 \\ 2\end{array}\right)$ conditions. Since

$$
\# \mathcal{T}_{2}= \begin{cases}3 \ell^{2}-3 \ell, & r=4 \ell+1, \\ 3 \ell^{2}+3 \ell+1, & r=4 \ell+2, \\ 3 \ell^{2}+3 \ell, & r=4 \ell+3, \\ 3 \ell^{2}+9 \ell+4, & r=4 \ell+4,\end{cases}
$$

the dimension of $\mathcal{S}_{r}\left(T_{\mathrm{PS} 6}\right)$ in each of the four cases is bounded below by the same quantities appearing in (4.5), which implies that $\operatorname{dim} \mathcal{S}_{r}\left(T_{\mathrm{PS} 6}\right)$ is given by the formula in (4.2).

To complete the proof, we need to show that $\mathcal{M}$ is a determining set and that it is stable. Suppose we fix $\left\{c_{\xi}\right\}_{\xi \in \mathcal{M}}$. We now show how to use the smoothness conditions to stably compute all other coefficients. For each $i=1,2,3$, we use the $C^{\mu}$ smoothness at $w_{i}$ to stably compute the coefficients corresponding to all other domain points in $D_{\mu}\left(w_{i}\right)$. Next, consider the sets of domain points

$$
L_{j}^{i}:=R_{d-j}\left(v_{T}\right) \cap\left\langle v_{T}, w_{i+1}, w_{i+2}\right\rangle, \quad j=0, \ldots, d,
$$

for $i=1,2,3$. For each $0 \leq j \leq r, L_{j}^{i}$ contains exactly $r$ domain points for which the corresponding coefficients have not yet been determined. The $C^{r}$ smoothness across the edge $\left\langle u_{i}, v_{T}\right\rangle$ gives $r$ univariate smoothness conditions involving these coefficients, and they can thus be determined from Lemma 2.1. At this point the proof divides into four cases.

Case 1. $r=4 \ell+1$. For each $j=1, \ldots, \ell$ and $i=1,2,3$, there are $r+2 j-2$ domain points on $R_{\mu+j}\left(w_{i}\right)$ whose corresponding coefficients have not yet been determined. These coefficients are subject to $r+2 j-2$ smoothness conditions, which correspond to the $C^{r}$ smoothness conditions combined with the $2 j-2$ functionals in $\mathcal{T}_{2}$. We can again get these coefficients from Lemma 2.1. Now for each $r+1 \leq j \leq r+\ell$ and $i=1,2,3$, the set $L_{j}^{i}$ contains $r$ domain points whose corresponding coefficients have not yet been computed. But then using the $C^{r}$ smoothness across the edge $\left\langle u_{i}, v_{T}\right\rangle$, Lemma 2.1 gives the values of these coefficients. To complete the proof in this case, we use Lemma 2.1 to perform the following cycle of computations: for each $j=1, \ldots, d-\mu-\ell$ :

a) compute the $r$ unset coefficients on the ring $R_{\mu+\ell+j}\left(w_{i}\right)$ for $i=1,2,3$,

b) compute the $r-2 j$ unset coefficients on $L_{r+\ell+j}^{i}$ for $i=1,2,3$.

This cycle of computations gives all the remaining coefficients.

Case 2. $r=4 \ell+2$. For each $j=1, \ldots, \ell$ and $i=1,2,3$ there are $r+2 j-1$ domain points on $R_{\mu+j}\left(w_{i}\right)$ whose corresponding coefficients have not yet been determined. These coefficients are subject to $r+2 j-1$ smoothness conditions obtained by combining the $C^{r}$ smoothness with the $2 j-1$ functionals in $\mathcal{T}_{2}$ corresponding to the set $\mathcal{E}_{2}$. We can thus compute these coefficients from Lemma 2.1. Now for each $r+1 \leq j \leq r+\ell$ and $i=1,2,3$, the set $L_{j}^{i}$ contains $r+1$ domain points whose corresponding coefficients have not yet been computed. But then using the $C^{r}$ smoothness across the edge $e:=\left\langle u_{i}, v_{T}\right\rangle$ together with the smoothness condition corresponding to $\tau_{e, r+j}^{r+1}$ in $\mathcal{T}_{2}$ with $e \in \mathcal{E}_{3}$, Lemma 2.1 gives these coefficients. 
Now for each $i=1,2,3$, we examine the ring $R_{\mu+\ell+1}\left(w_{1}\right)$, where we assume the edge $e:=\left\langle w_{1}, v_{T}\right\rangle$ is the edge chosen for $\widetilde{\mathcal{E}}_{2}$. There are $r+1$ domain points on this ring whose corresponding coefficients are not yet determined. Using the smoothness condition described by the functional $\tau_{e, \mu+\ell+1}^{r+1}$ in $\mathcal{T}_{2}$, we can use Lemma 2.1 to compute all of these coefficients. The lemma then gives the coefficients corresponding to domain points on the layers $L_{r+\ell+1}^{i}$ for $i=2,3$. We can now do the two rings $R_{\mu+\ell+1}\left(w_{2}\right)$ and $R_{\mu+\ell+1}\left(w_{3}\right)$, followed by the layer $L_{r+\ell+1}^{1}$. To complete the proof in this case, we use Lemma 2.1 to perform the following cycle of computations: for each $j=2, \ldots, d-\mu-\ell$ :

a) compute the $r-2 j+3$ unset coefficients on the layer $L_{\mu+\ell+j}^{i}$ for $i=1,2,3$,

b) compute the $r-1$ unset coefficients on the ring $R_{\mu+\ell+j}\left(w_{i}\right)$ for $i=1,2,3$.

This cycle of computations shows that all the remaining coefficients are determined.

The cases $r=4 \ell+3$ and $r=4 \ell+4$ can be handled in a similar way, and the proof is complete.

\section{Approximation POWER}

Let $\triangle$ be a triangulation of a polygonal domain $\Omega$, and let $\mathcal{S}_{r}\left(\triangle_{\mathrm{PS} 12}\right)$ be the macro-element space defined in (3.1). Let $|\triangle|$ be the mesh size of $\triangle$; i.e., the diameter of the largest triangle in $\triangle$. In this section we use the fact that $\mathcal{S}_{r}\left(\triangle_{\mathrm{PS} 12}\right)$ has a stable local minimal determining set $\mathcal{M}$ to show that the space has full approximation power. More precisely, we give bounds on how well functions $f$ in Sobolev spaces $W_{q}^{m+1}(\Omega)$ can be approximated in terms of $|\triangle|$ and the smoothness of $f$ as measured by the usual Sobolev semi-norm $|f|_{m+1, q, \Omega}$. Let

$$
\|g\|_{q, \Omega}:= \begin{cases}\left(\sum_{T \in \Delta}\|g\|_{q, T}^{q}\right)^{1 / q}, & 1 \leq q<\infty \\ \max _{T \in \Delta}\|g\|_{\infty, T}, & q=\infty\end{cases}
$$

Unless otherwise stated, all constants appearing in this section depend only on the smallest angle $\theta$ in the triangulation $\triangle_{\mathrm{PS} 12}$, or equivalently on the smallest angle in $\triangle$; see Remark 7.8. It is easy to see that $\left|\triangle_{\mathrm{PS} 12}\right| \leq|\triangle| / 2$.

Theorem 5.1. For all $f \in W_{q}^{m+1}(\Omega)$ with $1 \leq q \leq \infty$ and $m \leq d$, there exists a spline $s_{f} \in \mathcal{S}_{r}\left(\triangle_{\mathrm{PS} 12}\right)$ such that

$$
\left\|D_{x}^{\alpha} D_{y}^{\beta}\left(f-s_{f}\right)\right\|_{q, \Omega} \leq C|\triangle|^{m+1-\alpha-\beta}|f|_{m+1, q, \Omega},
$$

for all $0 \leq \alpha+\beta \leq m$. Here the constant $C$ depends only on the smallest angle in $\triangle$, and if $q<\infty$ also on the Lipschitz constant associated with the boundary of $\Omega$.

Proof. We give the proof only for $1 \leq q<\infty$. The case $q=\infty$ is similar and simpler. We begin by constructing a quasi-interpolant $Q$ mapping $L_{1}(\Omega)$ into the spline space $\mathcal{S}_{r}\left(\triangle_{\mathrm{PS} 12}\right)$. Fix $f \in L_{1}(\Omega)$. Then for each triangle $T \in \triangle_{\mathrm{PS} 12}$, we choose the largest disk contained in $T$, and let $F_{T}$ be the corresponding averaged Taylor polynomial of degree $d$ approximating $f$; see, e.g., [10. Then for each $\xi \in \mathcal{M} \cap T$, let $c_{\xi}:=\gamma_{\xi}\left(F_{T} f\right)$, where $\gamma_{\xi}$ is the linear functional that picks off the B-coefficient associated with the domain point $\xi$. We now define $Q f$ to be the spline in $\mathcal{S}_{r}\left(\triangle_{\mathrm{PS} 12}\right)$ whose other coefficients are determined from $\left\{c_{\xi}\right\}_{\xi \in \mathcal{M}}$ by using smoothness conditions as in the proof of Theorem 3.2. $Q$ is a linear projector mapping $L_{1}(\Omega)$ onto $\mathcal{S}_{r}\left(\triangle_{\mathrm{PS} 12}\right)$. 
Using the $L_{q}$ stability of the B-form and properties of $F_{T}$ (see [10]), we have

$$
\left|c_{\xi}\right|=\left|\gamma_{\xi}\left(F_{T} f\right)\right| \leq \frac{K_{1}}{A_{T}^{1 / q}}\left\|F_{T} f\right\|_{q, T} \leq \frac{K_{1} K_{2}}{A_{T}^{1 / q}}\|f\|_{q, T}, \quad \xi \in \mathcal{M} \cap T,
$$

where $A_{T}$ is the area of $T$. By the locality and stability of $\mathcal{M}$, it follows that if $\eta$ is a domain point lying in $T$, then

$$
\left|c_{\eta}\right| \leq \frac{K_{1} K_{2} K_{3}}{A_{\min }^{1 / q}}\|f\|_{q, \Omega_{T}}
$$

where $A_{\min }$ is the area of the smallest triangle in $\Omega_{T}:=\operatorname{star}^{3}(T)$. It is shown in [10] that the area of the largest triangle in $\Omega_{T}$ is bounded by a constant (depending only on $\theta$ ) times the area of the smallest triangle. Using the fact that the Bernstein basis polynomials form a partition of unity, we get $\|Q f\|_{q, T} \leq K_{4}\|f\|_{q, \Omega_{T}}$.

Now suppose $f \in W_{q}^{m+1}(\Omega)$ with $m \leq d$. Fix $0 \leq \alpha+\beta \leq m$ and $T \in \triangle_{\mathrm{PS} 12}$. Then using the Markov inequality [18, it follows that for any $p \in \mathcal{P}_{d}$,

$$
\begin{aligned}
\left\|D_{x}^{\alpha} D_{y}^{\beta}(f-Q f)\right\|_{q, T} & \leq\left\|D_{x}^{\alpha} D_{y}^{\beta}(f-p)\right\|_{q, T}+\left\|D_{x}^{\alpha} D_{y}^{\beta} Q(f-p)\right\|_{q, T} \\
& \leq\left\|D_{x}^{\alpha} D_{y}^{\beta}(f-p)\right\|_{q, T}+\frac{K_{5}}{\rho_{T}^{\alpha+\beta}}\|Q(f-p)\|_{q, T} \\
& \leq\left\|D_{x}^{\alpha} D_{y}^{\beta}(f-p)\right\|_{q, T}+\frac{K_{4} K_{5}}{\rho_{T}^{\alpha+\beta}}\|f-p\|_{q, \Omega_{T}},
\end{aligned}
$$

where $\rho_{T}$ is the diameter of the largest disk contained in $T$. It is shown in [10] that $\left|\Omega_{T}\right| \leq K_{6} \rho_{T}$. Now (cf. Lemma 4.6 of [10]), there exists a polynomial $p \in \mathcal{P}_{m}$ depending on $f$ with

$$
\left\|D_{x}^{i} D_{y}^{j}(f-p)\right\|_{q, \Omega_{T}} \leq K_{7}\left|\Omega_{T}\right|^{m+1-i-j}|f|_{m+1, q, \Omega_{T}},
$$

for all $0 \leq i+j \leq m$, where $K_{7}$ is a constant depending on $\theta$ and the Lipschitz constant of the boundary of $\Omega$. Inserting this in (5.2) leads to

$$
\begin{array}{r}
\left\|D_{x}^{\alpha} D_{y}^{\beta}(f-Q f)\right\|_{q, T} \leq K_{8}|\triangle|^{m+1-\alpha-\beta}|f|_{m+1, q, \Omega_{T}}, \\
\text { all } 0 \leq \alpha+\beta \leq m .
\end{array}
$$

Summing over all triangles $T \in \triangle_{\mathrm{PS} 12}$ and using the fact that the number of triangles in $\Omega_{T}$ is bounded by a constant depending only on $\theta$, we get (5.1).

\section{A NODAL DETERMINING SET FOR $\mathcal{S}_{r}\left(\triangle_{\mathrm{PS} 12}\right)$}

In this section we describe a nodal minimal determining set for $\mathcal{S}_{r}\left(\triangle_{\mathrm{PS} 12}\right)$ and a corresponding Hermite interpolation projector. For each triangle $T$ in $\triangle$, let $v_{T}$ be its barycenter. For each edge $e:=\langle u, v\rangle$ of $\triangle$, let $w_{e}$ be its midpoint, and let $w_{e}^{1}:=\frac{\mu u+(d-\mu) w_{e}}{d}$ and $w_{e}^{2}:=\frac{\mu v+(d-\mu) w_{e}}{d}$. Let $D_{e}$ be the directional derivative associated with a unit vector perpendicular to $e$. For each $i>0$, let

$$
\begin{aligned}
& \eta_{e, 1, j}^{i}:=\frac{(i-j+1) u+j w_{e}^{1}}{i+1}, \\
& \eta_{e, 2, j}^{i}:=\frac{(i-j+1) v+j w_{e}^{1}}{i+1}, \\
& \eta_{e, 3, j}^{i}:=\frac{(i-j+1) w_{e}^{1}+j w_{e}^{2}}{i+1},
\end{aligned}
$$


for $j=1, \ldots, i$. Finally, for any point $t \in \mathbb{R}^{2}$, let $\varepsilon_{t}$ be the point evaluation functional at $t$.

Theorem 6.1. The set

$$
\mathcal{N}:=\bigcup_{v \in \mathcal{V}} \mathcal{N}_{v} \cup \bigcup_{e \in \mathcal{E}}\left(\mathcal{N}_{e}^{1} \cup \mathcal{N}_{e}^{2} \cup \mathcal{N}_{e}^{3}\right)
$$

is a nodal determining set for $\mathcal{S}_{r}\left(\triangle_{\mathrm{PS} 12}\right)$, where

1) $\mathcal{N}_{v}:=\left\{\varepsilon_{v} D_{x}^{\alpha} D_{y}^{\beta}\right\}_{0 \leq \alpha+\beta \leq \rho}$,

2) $\mathcal{N}_{e}^{1}:=\bigcup_{i=1}^{\ell}\left\{\varepsilon_{\eta_{e, 1, j}^{i}} D_{e}^{\rho+\mu-d+1+i}\right\}_{j=1}^{i}$,

3) $\mathcal{N}_{e}^{2}:=\bigcup_{i=1}^{\ell}\left\{\varepsilon_{\eta_{e, 2, j}^{i}} D_{e}^{\rho+\mu-d+1+i}\right\}_{j=1}^{i}$,

4) $\mathcal{N}_{e}^{3}:=\bigcup_{i=1}^{m}\left\{\varepsilon_{\eta_{e, 3, j}^{i}} D_{e}^{i}\right\}_{j=1}^{i} \cup \bigcup_{i=1}^{m+1}\left\{\varepsilon_{\eta_{e, 3, j}^{i}} D_{e}^{\mu-i+1}\right\}_{j=1}^{i}$,

with $m=(\mu-1) / 2$.

Proof. It is easy to check that the cardinality of $\mathcal{N}$ is equal to the dimension of $\mathcal{S}_{r}\left(\triangle_{\mathrm{PS} 12}\right)$ as given in Theorem 3.2. Thus, it suffices to show that $\mathcal{N}$ is a nodal determining set; i.e., fixing $\{\lambda s\}_{\lambda \in \mathcal{N}}$ determines all coefficients of $s$. For every vertex $v$ of $\triangle$, we can compute all coefficients corresponding to domain points in the disk $D_{\rho}(v)$ directly from the data $\{\lambda s\}_{\lambda \in \mathcal{N}_{v}}$.

Given an edge $e$ of $\triangle$, let $w_{e}$ be its midpoint. We now compute all coefficients of $s$ corresponding to domain points in $D_{\mu}\left(w_{e}\right)$. By the $C^{\mu}$ smoothness at $w_{e}$, these coefficients can be regarded as the coefficients of a polynomial $g$ of degree $\mu$. Suppose we represent this polynomial in B-form relative to the triangle $\widetilde{T}:=\left\langle v_{e}, w_{e}^{1}, w_{e}^{2}\right\rangle$, where $v_{e}:=\left(\mu v_{T_{e}}+(d-\mu) w_{e}\right) / d$ and $v_{T_{e}}$ is the center of some triangle $T_{e}$ containing the edge $e$. As in Lemma 4.1, we can immediately compute the coefficients of $g$ in the disks $D_{m}\left(w_{e}^{1}\right) \cap \mathcal{D}_{\mu, \widetilde{T}}$ and $D_{m}\left(w_{e}^{2}\right) \cap \mathcal{D}_{\mu, \widetilde{T}}$. For each $i=1, \ldots, \mu$, we now compute the coefficients of $g$ corresponding to the remaining domain points on $R_{\mu-i}\left(v_{e}\right) \cap \mathcal{D}_{\mu, \widetilde{T}}$ from the derivative information given in 4$)$. We can now get the coefficients of $s$ corresponding to domain points in $D_{\mu}\left(w_{e}\right) \cap T_{e}$ by applying subdivision to $\widetilde{T}$. If $e$ is an interior edge, the coefficients of $s$ corresponding to the remaining domain points in $D_{\mu}\left(w_{e}\right)$ can be computed from the $C^{\mu}$ smoothness at $w_{e}$.

Next for each edge $e$, we use the values $\{\lambda s\}_{\lambda \in \mathcal{N}_{e}^{1}}$ to compute all coefficients of $s$ corresponding to domain points in $\mathcal{M}_{e}^{1}$. First, we consider $i=1$ in the definition of $\mathcal{M}_{e}^{1}$, i.e., the domain point $\xi_{\rho+\mu-d+2, d-\rho-1, d-\mu-1}^{T_{e}^{1}}$. This coefficient is determined by $D_{e, 1}^{\rho+\mu-d+2} s\left(\eta_{e, 1,1}^{1}\right)$, since all other coefficients involved in this derivative have already been computed. Then assuming we have dealt with the points in $\mathcal{M}_{e}^{1}$ up to $i-1$, we can use the values $\left\{D_{e, 1}^{\rho+\mu-d+1+i} s\left(\eta_{e, 1, j}^{i}\right)\right\}_{j=1}^{i}$ to find the coefficients corresponding to $\left\{\xi_{\rho+\mu-d+i+1, d-\rho-j, d-\mu-i+j-1}^{T_{e}^{1}}\right\}_{j=1}^{i}$. This involves solving an $i \times i$ linear system. A similar argument leads to the coefficients of $s$ corresponding to domain points in $\mathcal{M}_{e}^{2}$.

At this point we have determined all coefficients corresponding to domain points in the minimal determining set $\mathcal{M}$ of Theorem 3.2 and it follows from that theorem that all other coefficients are also determined. 
Theorem 6.1 shows that for any function $f \in C^{\mu}(\Omega)$, there is a unique spline $s \in \mathcal{S}_{r}\left(\triangle_{\mathrm{PS} 12}\right)$ solving the Hermite interpolation problem $\lambda s=\lambda f$ for all $\lambda \in \mathcal{N}$. The mapping that takes functions $f \in C^{\mu}(\Omega)$ to this Hermite interpolating spline defines a linear projector $\mathcal{I}$ mapping $C^{\mu}(\Omega)$ onto $\mathcal{S}_{r}\left(\triangle_{\mathrm{PS} 12}\right)$. We now give an error bound for how well $\mathcal{I} f$ approximates smooth functions $f$ in the maximum norm. We write $|\triangle|$ for the mesh size of the initial triangulation $\triangle$ before applying the Powell-Sabin-12 splits.

Given a triangle $T \in \triangle$ and a domain point $\xi \in T$ of $\mathcal{S}_{r}\left(\triangle_{\mathrm{PS} 12}\right)$, it is easy to see that if the coefficient $c_{\xi}$ of $\mathcal{I} f$ is computed from derivatives as in the proof of Theorem 6.1, then

$$
\left|c_{\xi}\right| \leq K_{1} \sum_{\nu=0}^{\mu}|T|^{\nu}|f|_{\nu, T}
$$

where $K_{1}$ is a constant depending only on the smallest angle in $\triangle$. Since the computation of all other coefficients from smoothness conditions (cf. the proofs of Theorems 3.2 and 6.1 is a stable process, it follows that 6.2 holds for all domain points $\xi$ lying in $T$. Since the Bernstein basis polynomials form a partition of unity, (6.2) implies

$$
\|\mathcal{I} f\|_{T} \leq K_{1} \sum_{\nu=0}^{\mu}|T|^{\nu}|f|_{\nu, T}
$$

Theorem 6.2. There exists a constant $K$ depending only on the smallest angle in $\triangle$ such that for every $f \in C^{m+1}(\Omega)$ with $\mu-1 \leq m \leq d$,

$$
\left\|D_{x}^{\alpha} D_{y}^{\beta}(f-\mathcal{I} f)\right\|_{\Omega} \leq K|\triangle|^{m+1-\alpha-\beta}|f|_{m+1, \Omega},
$$

for all $0 \leq \alpha+\beta \leq m$.

Proof. Fix $f \in C^{m+1}(\Omega)$ and a triangle $T \in \triangle$. Then Lemma 4.6 in [10] implies that there exists a polynomial $p \in \mathcal{P}_{m}$ such that

$$
\left\|D_{x}^{i} D_{y}^{j}(f-p)\right\|_{T} \leq K_{2}|T|^{m+1-i-j}|f|_{m+1, T}
$$

for all $0 \leq i+j \leq m$. Now fix $0 \leq \alpha+\beta \leq m$. Then since $\mathcal{I}$ reproduces polynomials, we have

$$
\left\|D_{x}^{\alpha} D_{y}^{\beta}(f-\mathcal{I} f)\right\|_{T} \leq\left\|D_{x}^{\alpha} D_{y}^{\beta}(f-p)\right\|_{T}+\left\|D_{x}^{\alpha} D_{y}^{\beta} \mathcal{I}(f-p)\right\|_{T},
$$

and to complete the proof it suffices to estimate the second term. Let $T_{1}, \ldots, T_{12}$ be the subtriangles in the Powell-Sabin-12 split of $T$. Then using the Markov inequality (cf. [10, 18]), it follows that

$$
\left\|D_{x}^{\alpha} D_{y}^{\beta} \mathcal{I}(f-p)\right\|_{T_{j}} \leq \frac{K_{3}}{\rho_{T_{j}}^{\alpha+\beta}}\|\mathcal{I}(f-p)\|_{T_{j}} \leq \frac{K_{1} K_{3}}{\rho_{T_{j}}^{\alpha+\beta}} \sum_{\nu=0}^{\mu}|T|^{\nu}|f-p|_{\nu, T}
$$

for all $j=1, \ldots, 12$, where $\rho_{T_{j}}$ is the diameter of the largest disk contained in $T_{j}$. By the geometry of the Powell-Sabin-12 split, $|T| \leq K_{4} \rho_{T_{j}}$, and taking the maximum over all $T \in \triangle$, we immediately get (6.4). 


\section{REMARKS}

Remark 7.1. We were first motivated to construct a family of smooth macroelements on the Powell-Sabin-12 split after hearing a lecture by Rong-Qing Jia in which he used a mixture of $C^{1}$ Powell-Sabin-6 and Powell-Sabin-12 elements in order to construct continuously differentiable wavelets on triangulations; see 8 .

Remark 7.2. It was shown in [5, 12] that it is not possible to construct $C^{r}$ macroelements on the Powell-Sabin-6 split using splines of lower degree than those considered here. Here we have constructed our macro-elements on Powell-Sabin-12 splits with the same degrees for the purposes of compatibility; cf. Remark 7.1. However, due to the special geometry of the Powell-Sabin-6 split of the triangle $\left\langle w_{1}, w_{2}, w_{3}\right\rangle$ inside the Powell-Sabin-12 split (see Definition 3.1 and Figure 4), we have found that it is possible to construct macro-elements in the Powell-Sabin-12 case with lower degrees. We plan to report on this elsewhere.

Remark 7.3. The Powell-Sabin-12 split was introduced in [14, where it was used to define a $C^{1}$ macro-element based on quadratic splines. This corresponds to our element for $r=1$. In this case the macro-element space has dimension 12, and the nodal degrees of freedom consist of the values and gradients at the three vertices of $T$ along with one cross-boundary derivative at the midpoint of each edge; see Figure 2,

Remark 7.4. The $C^{r}$ macro-elements constructed in [5, 12] provide global $C^{r}$ smoothness for a triangulation $\triangle$ which has been refined with Powell-Sabin-6 splits only if for each interior edge $e$ of $\triangle$, the split point $w_{e}$ on the edge $e$ lies on the line joining the interior points $v_{T}$ and $\tilde{v}_{T}$ of the two triangles $T$ and $\widetilde{T}$ that share $e$, and thus in general, $w_{e}$ will not be at the midpoint of $e$. This geometric constraint is not required for our Powell-Sabin-12 macro-elements.

Remark 7.5. In developing the macro-element spaces of this paper, we have made extensive use of the java code of Alfeld for examining determining sets for superspline spaces. The code is described in [1] and can be used or downloaded from http://www.math.utah.edu/ alfeld. The code not only checks whether a given set of domain points is a MDS, but also produces the equations needed to compute all unset coefficients from those that have been set.

Remark 7.6. The construction described here is not unique in the sense that there are other choices of the extra smoothness conditions which also lead to macroelements based on the degrees of freedom used here.

Remark 7.7. Frequently in practice one has to interpolate given values at scattered data points where no derivative information is provided. In this case, macro-element methods can still be applied, but the needed derivatives (or the equivalent set of B-coefficients) have to be estimated from the data.

Remark 7.8. Simple trigonometry shows that if $T_{\mathrm{PS} 12}$ is the Powell-Sabin split of a triangle $T$, then $\sin \left(\theta_{\mathrm{PS}}\right) \geq \sin (\theta) / 3$, where $\theta_{\mathrm{PS}}$ is the smallest angle in $T_{\mathrm{PS} 12}$ and $\theta$ is the smallest angle in $T$.

Remark 7.9. In [3] it was noted that the classical $C^{1}$ Clough-Tocher and PowellSabin macro-elements have natural analogs in terms of spherical splines. Since the algebra of spherical splines is essentially the same as for bivariate splines [2], 
it is clear that the entire family of macro-elements constructed here can also be immediately carried over to the sphere.

\section{REFERENCES}

[1] Alfeld, P., Bivariate spline spaces and and minimal determining sets, J. Comput. Appl. Math. 119 (2000), 13-27. MR.1774208(2001e:41013)

[2] Alfeld, Peter, Marian Neamtu, and Larry L. Schumaker, Dimension and local bases of homogeneous spline spaces, SIAM J. Math. Anal. 27(5) (1996), 1482-1501. MR1402451(97h:41059)

[3] Alfeld, Peter, Marian Neamtu, and Larry L. Schumaker, Fitting scattered data on spherelike surfaces using spherical splines, J. Comput. Appl. Math. 73 (1996), 5-43. MR1424867 (98a:41013)

[4] Alfeld, P. and L. L. Schumaker, Smooth macro-elements based on Clough-Tocher triangle splits, Numer. Math. 90 (2002), 597-616. MR1888831(2003a:65098)

[5] Alfeld, P. and L. L. Schumaker, Smooth macro-elements based on Powell-Sabin triangle splits, Advances in Comput. Math. 16 (2002), 29-46. MR.1888218 (2003a:65097)

[6] Alfeld, P. and L. L. Schumaker, Upper and lower bounds on the dimension of superspline spaces, Constr. Approx. 19 (2003), 145-161. MR1938936 (2003k:41010)

[7] Ibrahim, A. and L. L. Schumaker, Super spline spaces of smoothness $r$ and degree $d \geq 3 r+2$, Constr. Approx. 7 (1991), 401-423. MR.1120412 (92k:41017)

[8] Jia, R.-Q., Continuously differentiable wavelets on triangulations, in preparation.

[9] Lai, M.-J., On $C^{2}$ quintic spline functions over triangulations of Powell-Sabin's type, J. Comput. Appl. Math. 73 (1996), 135-155. MR1424873 (98a:41002)

[10] Lai, M.-J. and L. L. Schumaker, On the approximation power of bivariate splines, Advances in Comput. Math. 9 (1998), 251-279. MR.1662290 (2000b:41010)

[11] Lai, M.-J. and L. L. Schumaker, Macro-elements and stable local bases for splines on CloughTocher triangulations, Numer. Math. 88 (2001), 105-119. MR1819391 (2001k:65027)

[12] Lai, M.-J. and L. L. Schumaker, Macro-elements and stable local bases for splines on PowellSabin triangulations, Math. Comp. 72 (2003), 335-354. MR1933824(2003i:65012)

[13] Lai, M.-J. and L. L. Schumaker, Quadrilateral macroelements, SIAM J. Math. Anal. 33 (2002), 1107-1116. MR1897704 (2002k:41011)

[14] Powell, M. J. D. and M. A. Sabin, Piecewise quadratic approximations on triangles, ACM Trans. Math. Software 3 (1977), 316-325. MR0483304 (58:3319)

[15] Sablonnière, P. and Laghchim-Lahlou, M., Eléments finis polynomiaux composés de classe $C^{r}$, C. R. Acad. Sci. Paris 316, Série I (1993), 503-508. MR1209275 (94a:65059)

[16] Schumaker, L. L., Dual bases for spline spaces on cells, Comput. Aided Geom. Design 5 (1988), 277-284. MR0983463 (90a:41013)

[17] Schumaker, L. L., On super splines and finite elements, SIAM J. Numer. Anal. 26 (1989), 997-1005. MR:1005521 (90g:65016)

[18] Wilhelmsen, D. R., A Markov inequality in several dimensions, J. Approx. Theory 11 (1974), 216-220. MR0352826 (50:5312)

[19] Ženišek, A., Interpolation polynomials on the triangle, Numer. Math. 15 (1970), 283-296. MR0275014 (43:772)

Department of Mathematics, Vanderbilt University, Nashville, Tennessee 37240

E-mail address: s@mars.cas.vanderbilt.edu

Department of Mathematics, The University of Georgia, Athens, Georgia 30602 